\title{
Olive oil in the prevention and treatment of osteoporosis after artificial menopause
}

\author{
This article was published in the following Dove Press journal: \\ Clinical Interventions in Aging \\ 2 December 2014 \\ Number of times this article has been viewed
}

\section{Huilan Liu* \\ Huijuan Huang* \\ Boheng Li \\ Dong Wu \\ Fengmei Wang \\ Xiao hua Zheng \\ Qingxia Chen \\ Bifang Wu \\ Xiaojie Fan}

Department of Obstetrics and Gynecology, East Hospital, Xiamen University, Fuzhou, Fujian, People's Republic of China

*These authors contributed equally to this work
Correspondence: Huijuan Huang Department of Obstetrics and Gynecology, East Hospital, Xiamen University, The West Second Ring North Road No I 56 Fuzhou, Fujian 350025, People's Republic of China Email hhj352@।63.com
Purpose: The goal of this study was to investigate the anti-osteoporosis effect of extra virgin olive oil (EVOO) in vivo, and explore its antioxidant, anti-inflammatory properties in Sprague Dawley rats and its anticancer properties in patients.

Materials and methods: A total of 120 healthy female Sprague Dawley rats aged 6 months were divided into four groups: 1) sham-operated control (Sham group, $\mathrm{n}=30$ ); 2) ovariectomized (OVX group, $n=30) ; 3$ ) ovariectomized rats supplemented with EVOO (OVX + Olive, $n=30) ; 4$ ) ovariectomized rats supplemented with estrogen $\left(\mathrm{OVX}+\mathrm{E}_{2}, \mathrm{n}=30\right)$. EVOO and estrogen were administered by oral gavage at a dose of $1 \mathrm{~mL} / 100 \mathrm{~g}$ weight on a daily basis for 12 consecutive weeks. Twelve weeks later blood samples were obtained to detect the levels of calcium, alkaline phosphatase, phosphorus, interleukin-6 (IL-6), malonyldialdehyde (MDA), and nitrate content. Dual energy X-ray absorptiometer measured bone mineral density (BMD) of ovariectomized Sprague Dawley rats that had been fed olive oil for 3 months. Blood samples from patients, who regularly consumed olive oil over a 1 year period were also used to measure carbohydrate antigen 125 , carcino-embryonic antigen, $\alpha$-fetoprotein, and carbohydrate antigen 19-9 levels. BMD of lumbar spine and left femur was also evaluated by dual energy X-ray absorptiometry.

Results: Animal experiments showed that EVOO significantly increased BMD and decreased phosphatase, alkaline phosphatase, IL-6, MDA, and nitrate levels. However, it had no significant effect on the $\mathrm{Ca}^{2+}$ level. In clinical follow-up, EVOO also improved patient BMD levels on L3, L4, and left femoral neck, and reduced carbohydrate antigen 125, $\alpha$-fetoprotein, and carcino-embryonic antigen levels. But it had no significant effect on the carbohydrate antigen 19-9 level.

Conclusion: EVOO illustrated significant anti-osteoporosis, antioxidant, anti-inflammatory, and anticancer properties in vivo. However, further studies are required to determine the active component(s) responsible for these effects.

Keywords: olive oil, prevention, treatment, osteoporosis, artificial menopause

\section{Introduction}

Ovarian cancer, cervical cancer, and endometrial cancer are the three most common cancers of the female genital tract. The prevalence of these cancers is increasing and the age at onset has become younger in recent years - especially regarding ovarian cancer, with a mortality rate that ranks first out of all the abovementioned cancers. ${ }^{1}$ Surgery is the most important treatment for these cancers. However, an ovariectomy of both sides will lead to a sharp decline of estrogen, and the reduction of sex hormones at the time of menopause is known to increase the risk of osteoporosis. ${ }^{2,3}$ Meanwhile, the adjuvant chemotherapy after surgery may also induce bone loss which could cause a fracture and seriously affect the patient's quality of life. ${ }^{4}$ Currently, hormone replacement therapy (HRT) is widely used for osteoporosis after menopause. ${ }^{5}$ Although HRT may lower the rate of osteoporosis and fracture, large scale clinical research has shown that HRT was a factor in the growth of ovarian cancer ${ }^{6}$ and increased the incidence of breast 
cancer, cardiovascular disease, and stroke. Therefore, it is imperative to seek a safer and more effective therapy.

Olive oil plays a well-established, beneficial role in promoting good health. ${ }^{7}$ The International Olive Oil Council has defined the classification of virgin olive oil as follows: extra virgin olive oil (EVOO), virgin olive oil, and ordinary virgin olive oil. Oil retains the compounds that the fruit develops in response to environmental stress, especially phenolic compounds. ${ }^{8}$ Moreover, virgin olive oil is an integral ingredient of a Mediterranean diet, and its nutritional, medical, and cosmetic benefits are widely known and approved. ${ }^{9}$ Literature reviews show that in Europe, osteoporosis rarely happened in the Mediterranean region, ${ }^{10,11}$ as the traditional Mediterranean diet always has a high intake of olive oil. ${ }^{12}$ Indeed, virgin olive oil has been used as a folk remedy for combating diseases due to its hypotensive, cardioprotective, antimicrobial, anti-hyperglycemic, anticancer, and antiinflammatory pharmacological properties. ${ }^{13-16}$ Nowadays, epidemiological studies draw an important conclusion that virgin olive oil could release chronic diseases such as cardiovascular disease, ${ }^{17}$ atherosclerosis, ${ }^{18}$ and some types of cancers - especially colorectal and breast cancer. ${ }^{19}$ Olive oil, and its major component oleuropein, not only aids in the reduction of bone loss to help prevent osteoporosis, ${ }^{20}$ but its antioxidant, antiinflammatory function also acts to provide anti-endometrial ${ }^{21}$ and anti-breast cancer benefits, ${ }^{22}$ while protecting the cardiovascular system ${ }^{23}$ and benefiting hypoglycemic patients.

EVOO is obtained from the fruit of the olive tree solely by mechanical or physical means (the cold-pressed technique), which does not change the physical and chemical properties of the oil. Taking into account that EVOO contains many more phenolic compounds than the other oils (virgin olive oil [VOO], origin virgin olive oil [OVOO], olive oil [OO]), ${ }^{24}$ in this study we decided to investigate its anti-inflammatory, antioxidant, anticancer, and anti-osteoporosis effects by feeding olive oil to artificially induced menopausal Sprague Dawley rats. Additionally, ten patients were selected to evaluate olive oil's anti-osteoporosis and anti-cancer functions. EVOO illustrated significant antiosteoporosis, antioxidant, anti-inflammatory, and anticancer properties and may have broader clinical applications in the future.

\section{Materials and methods}

\section{Ethics statement}

All experimental procedures and protocols were approved by the Biomedical Ethics Committee of Xiamen University. All operations were performed under 5.2\% ketamine intraperitoneal injection anesthesia, and all efforts were made to minimize pain and discomfort.

\section{Chemicals}

EVOO (Earth Extra Virgin Olive Oil, PDO [Protected Designation of Origin], Greece) was purchased from a supermarket; diethylstilbestrol $0.5 \mathrm{mg}$ tablets (Chinese medical standard number H34021250), 5.2\% ketamine $(0.5 \mathrm{~mL} / 100 \mathrm{~g})$ (Chinese medical standard number 050405); alkaline phosphatase (ALP) kit (Sigma-Aldrich Co., St Louis, MO, USA); serum interleukin (IL)-6 assay kit (Sigma-Aldrich Co.).

\section{Animal experiments modeling approach and treatments}

All the animals were fed individually in Fuzhou General Hospital of Nanjing military region, under dry, ventilated, quiet, and fixed illumination conditions. The room temperature was $\sim 18^{\circ} \mathrm{C}-25^{\circ} \mathrm{C}, 50 \%-60 \%$ humidity, and the animals were allowed to eat and drink freely. After a week's adaptive growth, these rats were anesthetized using $5.2 \%$ amiodarone, then the skin in the center of the lower abdomen was cut vertically, opening the peritoneum to find the "Y"-shaped uterus. After identifying ovaries in the abdominal cavity, both ovaries were excised and sutured. Sham surgery ligation and excision of a piece of adipose tissue of the same size was done near the ovary and was medicated separately.

\section{Experimental animal grouping and treatment}

After an adaptation period of 7 days with a semi-purified standard diet ${ }^{25}$ thirty 6 month old female Sprague Dawley rats were divided into four groups: 1) Sham (sham-operated controls receiving the control diet); 2) OVX (ovariectomized controls also given the control diet); 3) OVX + Olive (ovariectomized rats given the control diet supplemented with $1 \mathrm{~mL} / 100 \mathrm{~g} /$ day EVOO); 4) $\mathrm{OVX}+\mathrm{E}_{2}$ (ovariectomized rats given the control diet mixed with $25 \mu \mathrm{g} / \mathrm{kg}$ diethylstilbestrol dissolved in phosphate-buffered saline). All rats were fed on a daily basis by way of gavage medication for 12 consecutive weeks.

\section{Measurement}

Index of serum metabolic profiles

Blood from the left ventricle was centrifuged at 2,500 rpm at $4^{\circ} \mathrm{C}$ for 10 minutes. The supernatant serum was aspirated into Eppendorf tubes and refrigerated at $-20^{\circ} \mathrm{C}$. When tested by automatic biochemical analyzer, the frozen serum was 
restored to room temperature. Plasma calcium $\left(\mathrm{Ca}^{2+}\right)$, phosphorus (P), and ALP expression levels were measured by routine methods. The plasma malonyldialdehyde (MDA) levels were determined according to the method of Draper and Hadley, ${ }^{26}$ based on the reaction of MDA with thiobarbituric acid. A double antibody sandwich method was used to detect serum IL levels, and measurements were conducted according to the steps of the kit.

\section{Bone density}

A dual energy X-ray bone density measuring instrument (Hologic, Bedford, MA, USA) (S/N806409); measured bone mineral density (BMD) of small animal's lumbar spine (L1, L2, L3, L4), and the left femur BMD; ${ }^{27}$ high resolution scan $(<0.11 \%)$ was used to measure rats' lumbar density adjusted resolution and the BMD of the left femur.

\section{Clinical data}

Ten patients aged 30-50 years who had undergone a hysterectomy and had bilateral ovarian and bilateral fallopian tube excised in obstetrics and gynecology were recruited and took olive oil beginning 1 month after surgery in Fuzhou General Hospital of Nanjing Military Region from January 2012 to January 2013. All of them were informed participants. None of them were menopausal before surgery. Pathology showed one ovarian cancer, five endometrial cancers, and four cervical cancers. Another ten patients with no menopause before surgery aged 30-50 years who also had undergone a hysterectomy and had bilateral ovarian and bilateral fallopian tube excised were chosen as a control group receiving no medication. Pathology after surgery showed two ovarian cancers, four endometrial cancers, and four cervical cancers. The experimental group took $50 \mathrm{~mL}$ of olive oil daily in the morning with no other medicine or treatment programs, but the control group took nothing in addition to their regular diet. Both the experimental and control groups had the same diet. A follow-up telephone call was made each week instructing the experimental group to take $50 \mathrm{~mL}$ of olive oil once every morning. All participants were asked about disease history and each participant received a thorough physical examination. Patients with diseases such as hyperthyroidism, rheumatoid arthritis, and diabetes were excluded from the study, as these diseases would affect bone metabolism. Moreover, patients in this research could not be bedridden, use any bone metabolism medicine, have any serious liver and kidney diseases, nor have a history of alcoholism or cigarette smoking.

\section{t-score}

The World Health Organization (WHO) provided criteria for diagnosing osteoporosis by dual energy X-ray scan. The BMD measurement of the patient (preferably the femoral neck, total hip, and lumbar spine) was compared with the mean BMD of a young, healthy cohort of females to produce a $t$-score. The $t$-score categories are normal ( $t$-score: $\geq$ -1.0 ), low bone mass (formerly called osteopenia, $t$-score: $>$ $-2.5 \sim<-1)$, and osteoporosis ( $t$-score: $\leq-2.5)$. Only the $t$-score was used for the purpose of diagnosing osteoporosis in postmenopausal women. ${ }^{28}$

\section{Statistical analysis}

All data are expressed as the mean \pm standard deviation (SD). The statistical significance of differences between controls and test values was assessed with variance analysis or LSD. $t$-test was used for comparison. Differences were considered significant if $P<0.05$.

\section{Results}

\section{Serum metabolites indexes and bone density changes of animal subjects $\mathrm{Ca}^{2+}$ and $\mathrm{P}$}

Through comparison of calcium among four different arrays, $F=0.952, P>0.05$, the differences had no statistical significance. That meant that $\mathrm{Ca}^{2+}$ levels did not obviously change in the four groups (Table 1). On the contrary, the P level markedly decreased in the OVX + Olive group, OVX group, and $\mathrm{OVX}+\mathrm{E}_{2}$ group when compared to the Sham group, but there was no statistical significance in the OVX + Olive oil group, OVX group, and OVX $+\mathrm{E}_{2}$ group (Table 1).

Table I Concentration of serum Ca ${ }^{2+} / \mathrm{P} / \mathrm{ALP} / \mathrm{IL}-6$ in different groups at 12 weeks post-operation

\begin{tabular}{llllll}
\hline Group & $\mathbf{n}$ & $\mathbf{C a}^{2+}(\mathbf{m m o l} / \mathbf{L})$ & $\mathbf{P}(\mathbf{m m o l} / \mathbf{L})$ & ALP $(\mathbf{U} / \mathbf{L})$ & IL-6 $(\mathbf{n g} / \mathbf{m L})$ \\
\hline I Sham group & 30 & $2.82 \pm 0.02$ & $6.85 \pm 0.18$ & $184.1 \pm 10.4$ & $70.303 \pm 5.654$ \\
2 OVX group & 30 & $2.76 \pm 0.03$ & $4.84 \pm 0.12^{*}$ & $230.9 \pm 2.6^{*}$ & $128.812 \pm 7.216^{*}$ \\
3 OVX + Olive group & 30 & $2.77 \pm 0.02$ & $4.87 \pm 0.13^{*}$ & $202.9 \pm 2.6$ & $86.414 \pm 7.137$ \\
4 OVX $+\mathrm{E}_{2}$ group & 30 & $2.76 \pm 0.03$ & $4.91 \pm 0.13^{*}$ & $211.8 \pm 4.9$ & $90.362 \pm 5.293$ \\
\hline
\end{tabular}

Notes: Data represent the means \pm standard deviation (SD). *Means $P<0.05$, revealed the differences among those groups that were statistically significant compared with the sham group.

Abbreviations: ALP, alkaline phosphatase; OVX, ovariectomized group; IL, interleukin; $\mathrm{E}_{2}$, estrogen; P, phosphorus. 
Table 2 Concentration of serum MDA and nitrate in different groups at 12 weeks post-operation

\begin{tabular}{|c|c|c|c|c|c|}
\hline & $\begin{array}{l}\text { Sham group } \\
(n=30)\end{array}$ & $\begin{array}{l}\text { OVX group } \\
(n=30)\end{array}$ & $\begin{array}{l}\text { OVX + Olive } \\
\text { group }(n=30)\end{array}$ & $\begin{array}{l}\text { OVX }+E_{2} \\
\text { group }(n=30)\end{array}$ & P-value \\
\hline MDA ( $\mu \mathrm{mol} / \mathrm{L})$ & $1.23 \pm 0.38$ & $2.58 \pm 0.06$ & $1.76 \pm 0.04$ & $1.56 \pm 0.09$ & $0.021^{*}$ \\
\hline Nitrate $(\mu \mathrm{mol} / \mathrm{L})$ & $15.65 \pm 0.45$ & $21.23 \pm 0.36$ & $9.16 \pm 0.38$ & $|2.45 \pm 0.4|$ & $0.017^{*}$ \\
\hline
\end{tabular}

Notes: Data represent the means \pm standard deviation (SD). *Means $P<0.05$, revealed the differences among those groups that were statistically significant. Abbreviations: OVX, ovariectomized group; MDA, malonyldialdehyde; $\mathrm{E}_{2}$, estrogen.

\section{ALP and IL-6}

Both ALP and IL-6 levels were higher in group OVX than in the other three groups. OVX + Olive group and $\mathrm{OVX}+\mathrm{E}_{2}$ group showed no statistical differences between the two indexes, which suggested that olive oil and estrogen probably had the same effect on osteogenesis (Table 1).

\section{MDA and nitrate}

MDA and nitrate levels increased significantly in the OVX group, but in the OVX + Olive group MDA increased slightly, and nitrate notably decreased as reported by González-Correa et $\mathrm{al}^{29}$ that olive oil containing phenolic compounds might decrease inducible NO synthetase activity. ${ }^{25}$ These results indicated that olive oil probably played an important role in anti-oxidation to prevent bone loss (Table 2).

A

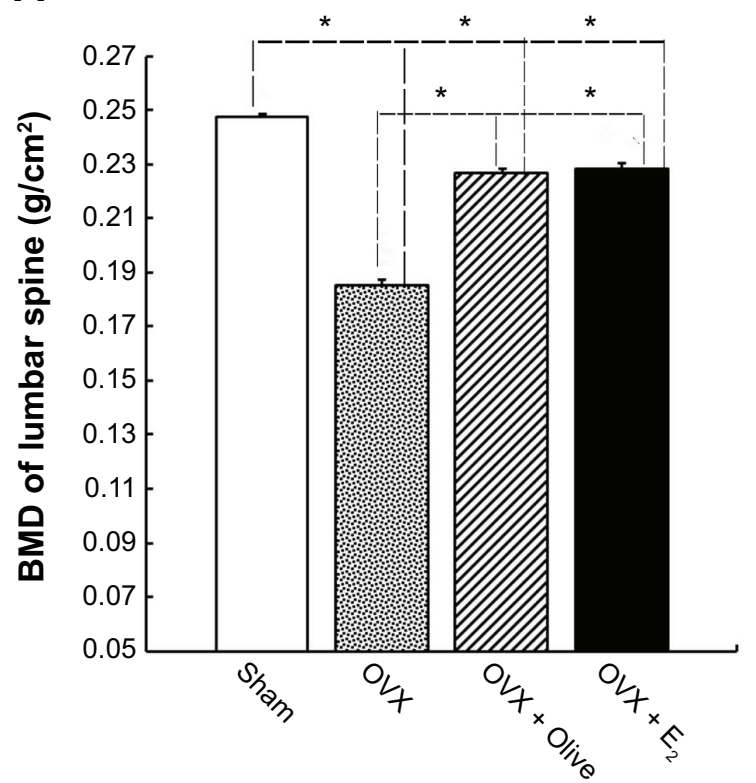

Bone density changes after 3 months' treatment

The average bone density of lumbar spine and left femur in Sham group were both higher than in the other three groups $(P<0.05)$ (Figure 1). Comparing OVX + Olive group with OVX $+\mathrm{E}_{2}$ group, $P>0.05$ (Figure 1), no significant differences were found. However, bone density of lumbar spine and left femur of OVX + Olive group and OVX $+\mathrm{E}_{2}$ group declined more slowly than OVX group $(P<0.05)$ (Figure 1). This implied that olive oil had a positive effect on preventing bone loss.

\section{The tumor markers and BMD changes of volunteers who did, or did not take olive oil regularly after surgery}

During the 1 month after their uterine and bilateral ovarian and bilateral fallopian tube were excised, those patients were

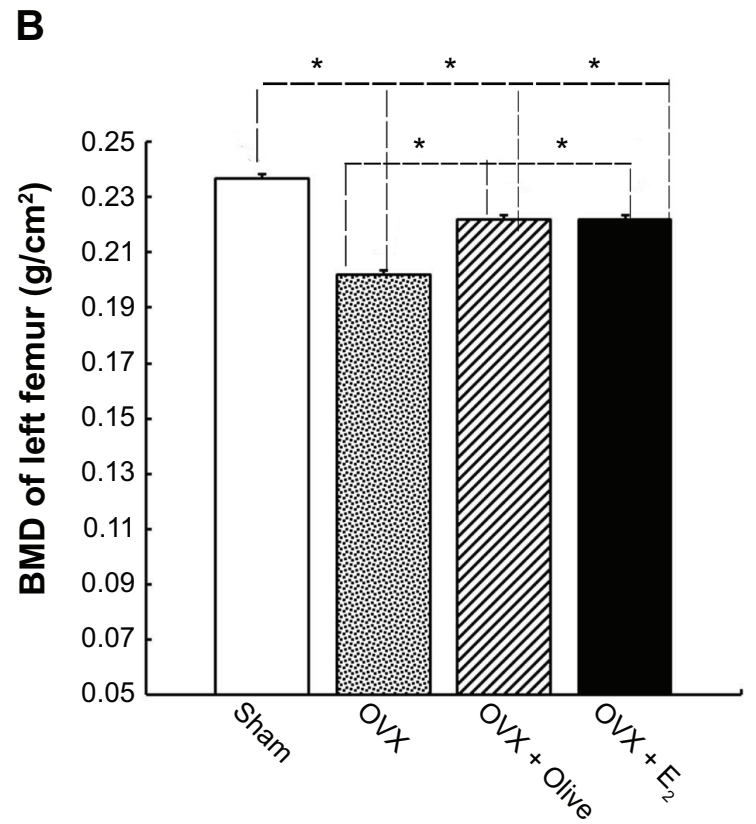

Figure I BMD of different groups at 12 weeks post-operation.

Notes: Comparison of bone density in Sham, OVX, OVX + Olive, OVX $+E_{2}$ groups at 12 weeks post-surgery. Data represent the means \pm standard deviation (SD) ( $n=30$ ). (A) and (B): OVX, OVX + Olive, OVX $+\mathrm{E}_{2}$ groups versus Sham group, bone density of lumbar spine and bone density of left femur were all lower (*P<0.05). Bone density of lumbar spine and bone density of left femur of OVX + Olive group and OVX $+\mathrm{E}_{2}$ group declined more slowly versus $\mathrm{OVX}$ group $(* P<0.05)$. OVX + Olive group and $\mathrm{OVX}+\mathrm{E}_{2}$ group showed no significant difference.

Abbreviations: $\mathrm{OVX}$, ovariectomized group; $\mathrm{BMD}$, bone mineral density; $\mathrm{E}_{2}$, estrogen. 
Table 3 Patients' physical conditions data and indexes before taking olive oil (mean \pm SD)

\begin{tabular}{|c|c|c|c|c|}
\hline & & $\begin{array}{l}\text { Experimental group } \\
(\text { mean } \pm \text { SD })(n=10)\end{array}$ & $\begin{array}{l}\text { Control group } \\
(\text { mean } \pm \text { SD) }(n=10)\end{array}$ & $P$-value \\
\hline Age (years) & & $46.70 \pm 9.097$ & $48.65 \pm 8.928$ & 0.921 \\
\hline Weight (kg) & & $55.72 \pm 7.29 \mid$ & $56.53 \pm 6.003$ & 0.608 \\
\hline Height (cm) & & $158.1 \pm 5.379$ & $|60.70 \pm 8.5| \mid$ & 0.329 \\
\hline CAI $25(\mathrm{U} / \mathrm{mL})$ & & $70.6 \pm 13.00$ & $72.25 \pm 13.872$ & 0.763 \\
\hline AFP (ng/mL) & & $7.51 \pm 1.996$ & $7.43 \pm 1.880$ & 0.821 \\
\hline CEA (ng/mL) & & $6.47 \pm 0.923$ & $6.35 \pm 0.897$ & 0.563 \\
\hline CAI9-9 $(\mu / \mathrm{mL})$ & & $24.7 \pm 8.396$ & $24.0 \pm 7.747$ & 0.644 \\
\hline \multirow[t]{3}{*}{$\operatorname{BMD}\left(\mathrm{g} / \mathrm{cm}^{2}\right)$} & L3 & $0.9906 \pm 0.0154$ & $0.9916 \pm 0.0164$ & 0.890 \\
\hline & L4 & $0.9784 \pm 0.0123$ & $0.9789 \pm 0.014$ & 0.933 \\
\hline & Left femur & $0.7726 \pm 0.010$ & $0.7731 \pm 0.012$ & 0.937 \\
\hline
\end{tabular}

Notes: Data represent the means \pm standard deviation (SD). No significant differences were found.

Abbreviations: CAI25, carbohydrate antigen 125; AFP, $\alpha$-fetoprotein; CEA, carcino-embryonic antigen; CAI9-9, carbohydrate antigen 19-9; BMD, bone mineral density.

involved in follow-up monitoring and were divided into the experimental group (ten cases) and the control group (ten cases). Before taking olive oil, the patients of the two groups were nearly the same age, weight, and height; and some serum indexes such as carbohydrate antigen 125 (CA125), carcino-embryonic antigen (CEA), $\alpha$-fetoprotein (AFP), carbohydrate antigen 19-9 (CA19-9), lumbar spine (L3, L4) BMD, left femur BMD (Table 3), and their $t$-score categories were normal (Table 4). After taking olive oil for 1 year, CA125, CEA, and AFP levels in the experimental group decreased considerably $(P<0.05)$. But CA19-9 had no obvious changes with comparison to control $(P>0.05)$ (Figure 2$)$. Interestingly, after 1 year in the experimental group, CA125, AFP, and CEA were markedly decreased compared to the control group $(P<0.05)$. However, CA19-9 showed no difference (Figure 2). And according to Figure 3, the BMD of L3, L4, and the left femur was maintained at the previous level. Meanwhile, $t$-score categories were normal in the experimental group that took olive oil for 1 year. But in the control group with no olive oil taken, BMD obviously decreased and $t$-score categories showed low bone mass compared with the experimental group $(P<0.05)$ (Figure 3 and Table 4$)$.

Table 4 Patients' $t$-score in control group and experimental group (mean \pm SD)

\begin{tabular}{llll}
\hline Score & $\begin{array}{l}\text { Experimental group } \\
(\mathbf{m e a n} \pm \mathbf{S D})(\mathbf{n}=\mathbf{l 0})\end{array}$ & $\begin{array}{l}\text { Control group } \\
(\text { mean } \pm \mathbf{S D})(\mathbf{n}=\mathbf{I 0})\end{array}$ & $P$-value \\
\hline$t$-score & $-0.518 \pm 0.059$ & $-0.510 \pm 0.058$ & 0.594 \\
$t$-score' & $-0.548 \pm 0.029$ & $-0.107 \pm 0.097$ & $0.0013^{*}$ \\
\hline
\end{tabular}

Notes: $t$-score means a scoring standard representative of patients who did not take olive oil before experiment. $t$-score' means a scoring standard representative of patients who did and did not take olive oil for I year. *Represents after I year, in control group, patients' $t$-scores considerably decreased compared with experimental group.

Abbreviation: SD, standard deviation.
This confirmed that olive oil would be a good substitute for estrogen as a bone loss treatment.

\section{Discussion}

In this study, results of animal tests showed that olive oil could obviously prevent BMD decline of ovariectomized Sprague Dawley rats. In addition, it could decrease IL-6, MDA, nitrate, ALP, and P serum levels and would not influence $\mathrm{Ca}^{2+}$ level. Following up with the clinical patients, olive oil also prevented BMD decline of patients' lumbar spines and left femurs and decreased CA125, AFP, and CEA levels, but had no effect on CA19-9.

$\mathrm{Ca}^{2+}$ and $\mathrm{P}$ are important components of the skeleton, as they participate in skeletal development. Enough $\mathrm{Ca}^{2+}$ and $\mathrm{P}$ can help prevent bone loss and lower the risk of fracture. Many researchers have demonstrated that $\mathrm{Ca}^{2+}$ and $\mathrm{P}$ are important for preventing osteoporosis and promoting the function of bone density. A recent research study indicated that after taking olive oil, the P solubility in the serum of ovariectomized rats was considerably lowered, which could be the basis for proving the function of olive oil in the cure of osteoporosis. ${ }^{30}$ Our experimental results confirmed this view. But the data from our research showed that after the ovary was excised, the blood $\mathrm{Ca}^{2+}$ of Sprague Dawley rats did not change noticeably, as did the ovariectomized rats'. These results are inconsistent with Saleh and Saleh's ${ }^{30}$ research, considering it is related to the blood $\mathrm{Ca}^{2+}$ fluctuation during the time of osteoporosis. Twelve weeks after the rats' castration, the osteoclasts and the osteogenesis were in a new dynamic equilibrium (plateau). This is similar to the results of calcium dynamic research: ${ }^{30}$ absolute bone resorption rate did not increase, though it exceeded the bone formation rate of 50 100 mg per day, and bone mass changed a little. Bone metabolism balance depends on the anabolism of osteoblasts 

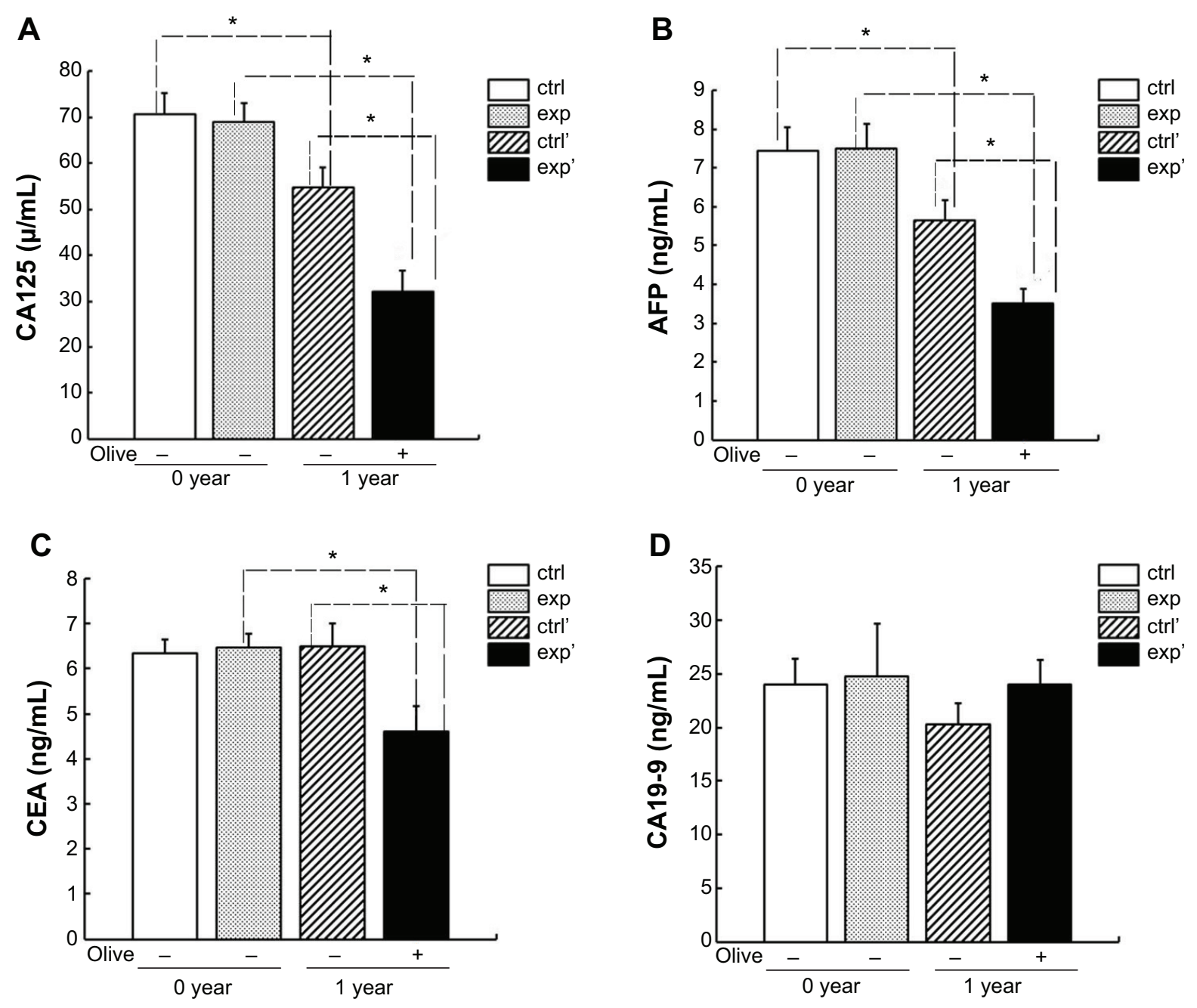

Figure 2 Comparison of tumor marker changes of the two followed-up patients.

Notes: CAI25, AFP, CEA, and CA 19-9 were examined in patients who did or did not take olive oil regularly after surgery. Data represents the means \pm standard deviation $(S D)(n=10)$. (A), (B), (C) revealed that after taking olive oil for I year in experimental group, serum CAI25, AFP, CEA considerably decreased versus "exp" group (*P $<0.05)$. Serum CEA of control group who did not take olive oil had not changed significantly after I year. After I year, in "exp"'group, CAI25, AFP, and CEA considerably decreased versus "ctrl" and versus "exp" $(* P<0.05)$. (D) CAI9-9 had no obvious changes with comparison to other groups $(P>0.05)$. "ctrl”" refers to patients who did not take olive oil for I year. "exp" refers to patients who took olive oil for I year.

Abbreviations: CAI25, carbohydrate antigen 125; AFP, $\alpha$-fetoprotein; CEA, carcino-embryonic antigen; CAI9-9, carbohydrate antigen 19-9; exp, experimental; ctrl, control.

and the catabolism of osteoclasts. Mineralization of osteoblasts plays an important role in osteoporosis. ALP is known as a marker of osteoblastic differentiation. ${ }^{31}$ Activated ALP promotes mineralization of matrix proteins by hydrolysis of pyrophosphate and inorganic P. ${ }^{32}$ This research compared the OVX + Olive group with the OVX group, and the statistical difference demonstrated that olive oil could lower the serum ALP solubility of castrated Sprague Dawley rats. In speculation, olive oil inhibits bone loss by lowering the ALP content which impairs mineralized matrix protein formation by osteogenesis cells. This mechanism needs further research.

Antioxidant systems exist in humans. They can remove reactive oxygen species. When the reactive oxygen species level increases and the activation of antioxidant systems decreases, the organism is in oxidation stress condition. Oxidation stress can lead to protein denaturation and lipid peroxidation. MDA is lipid peroxide caused by radicals attacking biomembrane polyunsaturated fatty acids. Therefore, the test of MDA can reflect the lipid peroxidation degree of the organism, which indirectly reflects the degree of cell damage. Oxidation stress and osteoblast apoptosis always accompanies osteoporosis. The probable reasons are the activation of osteoclast cells and the bone matrix degradation. ${ }^{33}$ In our study, the serum MDA level increased in the OVX group and obviously decreased in the OVX + Olive group, which implied that olive oil might lower the oxidation degree of lipids for the prevention of osteoporosis. Olive oil is rich in antioxidative fatty acids, which can decrease oxidative stress. ${ }^{34}$ Besides, after taking olive oil, the nitrate activity 

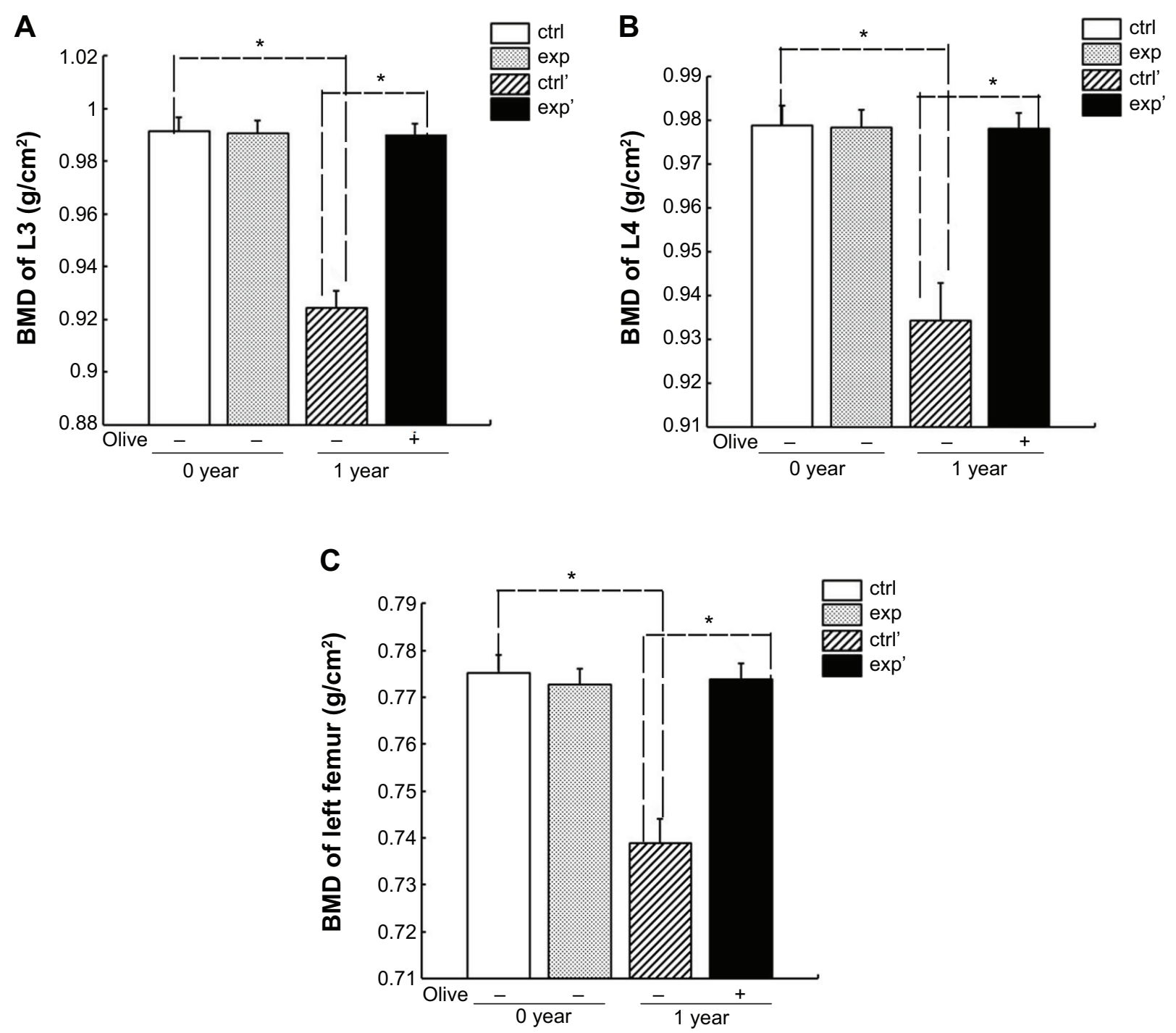

Figure 3 Comparison of BMD levels of followed-up patients.

Notes: BMD of L3, L4, and left femur was detected in patients who did or did not take olive oil regularly after surgery for I year. Data represent the means \pm standard deviation (SD) ( $n=10)$. $* P<0.05$ versus "ctrl”" and $* P<0.05$ versus "exp"'. "ctrl”" refers to patients who did not take olive oil for I year. "exp"” refers to patients who took olive oil for I year.

Abbreviations: BMD, bone mineral density; exp, experimental; ctrl, control.

and solubility in the plasma of castrated rats decreased significantly. This experiment showed that when comparing the OVX + Olive group with the OVX group, solubility of nitrate was greatly reduced in the OVX + Olive group. The result of our study is consistent with the report, but the reason is not clear. Further research needs to be conducted. Olive oil includes phenolic compounds which decrease inducible NO synthetase activity. ${ }^{25}$ Hydroxytyrosol was able to inhibit COX-2 and iNOS gene expression. ${ }^{27}$ Hydroxytyrosol can decrease the source of synthesis of nitrate.

In our research, IL-6 of OVX group is obviously higher than the other three groups. IL-6 mainly comes from lymphocytes. IL-6 overexpressed transgenic rats showed bone loss, microstructures of cortical and cancellous bones, a decrease of osteoblasts and vitality, and an increase of bone resorption. Recently, scholars think that the reason IL-6 initiates bone resorption is its induction of osteoclasts and interaction with RANKL-RANK-OPG axis. ${ }^{35,36}$ On one hand, IL-6 can increase RANKL expression of osteoblasts; on the other hand, IL-6 may exert its function on osteoclasts by tumor necrosis factor $\alpha$ and IL-1. Studies have shown that the antibody of IL- 6 can partly prevent the tumor necrosis factor $\alpha$ and IL-1 from inducing osteoclasts. ${ }^{31}$ Our previous study showed that the olive oil decreased serum IL-6 of animal models. ${ }^{32}$ Although studies have shown that oleuropein in olive oil lowers the RANKL expression of osteoblasts to inhibit osteoclast differentiation and maturation by reducing IL-1 inflammatory cytokine secretion, no articles have clarified the mechanism of decreasing IL-6 to prevent

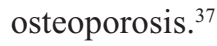


The main features of osteoporosis are progressive bone loss and degenerative bone microstructure. ${ }^{38}$ Bone metabolic balance depends on the anabolism of osteoblasts and the catabolism of osteoclast cells. The female's bone loss after menopause is related to estrogen deficiency. Main pathogenesis is that the estrogen deficiency causes osteoclast proliferation and differentiation. As the osteoclast cells' apoptosis decreases, the speed of bone resorption becomes faster than bone formation, which leads to osteoporosis. Whether the animal tests or followed-up patients are considered, results of both demonstrate that BMD decreased more slowly in the experimental group. So we speculated that olive oil prevents osteoporosis by inhibiting osteoclast growth, differentiation, and maturation.

The average AFP, CEA, and CA125 levels of the ten patients in the experimental group are lower than those of the control group and average BMD levels decreased more slowly than the control group, which indicated that EVOO has anti-osteoporosis and antitumor properties. A number of research projects have proved that these antitumor properties would prevent DNA damage, ${ }^{19}$ which plays an important role in osteoporosis. EVOO is rich in oleuropein which can inhibit the growth, movement, and attack of T-47D breast cancer cells, LN-18 nurogliocytoma cells, and melanoma cells. The aglycone, one of the decomposition products of oleuropein, can suppress breast cancer by inhibition of HER2 oncogene. ${ }^{21}$ Some studies have conjectured that the main reason tumor cells could stimulate osteoclast proliferation ${ }^{16}$ was because they expressed RANKL or RANK, or increased other immune cells' RANKL expression, or decreased OPG expression to activate the RANKL-RANK signaling pathway, ${ }^{17}$ which had been proven in prostate cancer. ${ }^{18}$ Therefore we conjecture that oleuropein could inhibit tumor formation by decreasing HER2 oncogene expression and activation, which is an effective way to cure osteoporosis.

In conclusion, olive oil can not only improve several serum bone indicators in castrated rats, but also prevent bone loss. Olive oil prevents bone loss in tumor patients and has excellent prospects for curing osteoporosis after menopause. A significantly greater number of patients should be studied in the future for a more persuasive result.

\section{Acknowledgment}

This work was supported by the military medical and technology innovation projects (11MA111).

\section{Disclosure}

The authors report no conflicts of interest in this work.

\section{References}

1. Lyman GH, Khorana AA, Kuderer NM, et al. Venous thromboembolism prophylaxis and treatment in patients with cancer: American Society of Clinical Oncology clinical practice guideline update. J Clin Oncol. 2013; 31(17):2189-2204.

2. Puel C, Mardon J, Kati-Coulibaly S, et al. Black Lucques olives prevented bone loss caused by ovariectomy and talc granulomatosis in rats. Br JNutr. 2007;97(5):1012-1020.

3. Ershler WB, Harman SM, Keller ET. Immunologic aspects of osteoporosis. Dev Comp Immunol. 1997;21(6):487-499.

4. Valls RM, Farras M, Suarez M, et al. Effects of functional olive oil enriched with its own phenolic compounds on endothelial function in hypertensive patients. A randomised controlled trial. Food Chem. 2015;167: $30-35$.

5. Stevenson JC, Panay N, Pexman-Fieth C. Corrigendum to Oral estradiol and dydrogesterone combination therapy in postmenopausal women: Review of efficacy and safety. Maturitas. 2013;76(4):388.

6. Collaborative Group on Epidemiological Studies of Ovarian Cancer; Beral V, Doll R, Hermon C, Peto R, Reeves G. Ovarian cancer and oral contraceptives: collaborative reanalysis of data from 45 epidemiological studies including 23,257 women with ovarian cancer and 87,303 controls. Lancet. 2008;371(9609):303-314.

7. Kontou N, Psaltopoulou T, Panagiotakos D, Dimopoulos MA, Linos A. The mediterranean diet in cancer prevention: a review. $J$ Med Food. 2011;14(10):1065-1078.

8. Guerfel M, Ben Mansour M, Ouni Y, Guido F, Boujnah D, Zarrrouk M. Triacylglycerols composition and volatile compounds of virgin olive oil from Chemlali cultivar: comparison among different planting densities. Scientific World Journal. 2012;2012:354019.

9. Berry EM, Arnoni Y, Aviram M. The Middle Eastern and biblical origins of the Mediterranean diet. Public Health Nutr. 2011;14(12A): 2288-2295.

10. Kontogianni MD, Melistas L, Yannakoulia M, Malagaris I, Panagiotakos DB, Yiannakouris N. Association between dietary patterns and indices of bone mass in a sample of Mediterranean women. Nutrition. 2009;25(2):165-171.

11. Rivas A, Romero A, Mariscal-Arcas M, et al. Mediterranean diet and bone mineral density in two age groups of women. Int J Food Sci Nutr. 2013;64(2):155-161.

12. Schwingshackl L, Hoffmann G. Adherence to Mediterranean diet and risk of cancer: a systematic review and meta-analysis of observational studies. Int J Cancer. 2014;135(8):1884-1897.

13. Tuck KL, Hayball PJ. Major phenolic compounds in olive oil: metabolism and health effects. J Nutr Biochem. 2002;13(11):636-644.

14. Al-Azzawie HF, Alhamdani MS. Hypoglycemic and antioxidant effect of oleuropein in alloxan-diabetic rabbits. Life Sci. 2006; 78(12):1371-1377.

15. De la Puerta R, Martinez-Dominguez E, Ruiz-Gutierrez V. Effect of minor components of virgin olive oil on topical antiinflammatory assays. Z Naturforsch C. 2000;55(9-10):814-819.

16. Ruiz-Canela M, Martinez-Gonzalez MA. Olive oil in the primary prevention of cardiovascular disease. Maturitas. 2011;68(3): 245-250.

17. Kok FJ, Kromhout D. Atherosclerosis - epidemiological studies on the health effects of a Mediterranean diet. Eur J Nutr. 2004;43 Suppl 1: $1 / 2-5$.

18. Pelucchi C, Bosetti C, Negri E, Lipworth L, La Vecchia C. Olive oil and cancer risk: an update of epidemiological findings through 2010. Curr Pharm Des. 2010;17:805-812.

19. Puel C, Mathey J, Agalias A, et al. Dose-response study of effect of oleuropein, an olive oil polyphenol, in an ovariectomy/inflammation experimental model of bone loss in the rat. Clin Nutr. 2006;25(5): 859-868.

20. García-Martínez O, Mazzaglia G, Sánchez-Ortiz A, Ocaña-Peinado FM, Rivas A. Phenolic content of Sicilian virgin olive oils and their effect on MG-63 human osteoblastic cell proliferation. Grasas y Aceites. 2014; 65(3):e032. 
21. Carrera-Gonzalez MP, Ramirez-Exposito MJ, Mayas MD, MartinezMartos JM. Protective role of oleuropein and its metabolite hydroxytyrosol on cancer. Trends in Food Science and Technology. 2013;31(2):92-99.

22. Granados-Principal S, Quiles JL, Ramirez-Tortosa CL, Sanchez-Rovira P, Ramirez-Tortosa MC. Hydroxytyrosol: from laboratory investigations to future clinical trials. Nutr Rev. 2010;68(4):191-206.

23. Mohagheghi F, Bigdeli MR, Rasoulian B, Zeinanloo AA, Khosbaten A. Dietary virgin olive oil reduces blood brain barrier permeability, brain edema, and brain injury in rats subjected to ischemia-reperfusion. Scientific World Journal. 2010;10:1180-1191.

24. Ramirez-Tortosa MC, Urbano G, Lopez-Jurado M, et al. Extra-virgin olive oil increases the resistance of LDL to oxidation more than refined olive oil in free-living men with peripheral vascular disease. $J$ Nutr. 1999;129(12):2177-2183.

25. Puel C, Quintin A, Agalias A, et al. Olive oil and its main phenolic micronutrient (oleuropein) prevent inflammation-induced bone loss in the ovariectomised rat. Br J Nutr. 2004;92(1):119-127.

26. Draper HH, Hadley M. Malondialdehyde determination as index of lipid peroxidation. Methods Enzymol. 1990;186:421-431.

27. Puel C, Mardon J, Agalias A, et al. Major phenolic compounds in olive oil modulate bone loss in an ovariectomy/inflammation experimental model. J Agric Food Chem. 2008;56(20):9417-9422.

28. Cosman F, de Beur SJ, Leboff MS, et al. Clinician's Guide to Prevention and Treatment of Osteoporosis. Osteoporos Int. 2014; 25(10):2359-2381.

29. Gonzalez-Correa JA, Munoz-Marin J, Arrebola MM, et al. Dietary virgin olive oil reduces oxidative stress and cellular damage in rat brain slices subjected to hypoxia-reoxygenation. Lipids. 2007;42(10):921-929.

30. Saleh NK, Saleh HA. Olive oil effectively mitigates ovariectomyinduced osteoporosis in rats. BMC Complement Altern Med. 2011;11:10.
31. Hagiwara K, Goto T, Araki M, Miyazaki H, Hagiwara H. Olive polyphenol hydroxytyrosol prevents bone loss. Eur J Pharmacol. 2011;662(1-3):78-84.

32. Wang W, Olson D, Liang G, et al. Collagen XXIV (Col24alpha1) promotes osteoblastic differentiation and mineralization through TGF-beta/ Smads signaling pathway. Int J Biol Sci. 2012;8(10):1310-1322.

33. Sanchez-Rodriguez MA, Ruiz-Ramos M, Correa-Munoz E, MendozaNunez VM. Oxidative stress as a risk factor for osteoporosis in elderly Mexicans as characterized by antioxidant enzymes. BMC Musculoskeletel Disord. 2007;8:124.

34. Moreno JJ, Carbonell T, Sanchez T, Miret S, Mitjavila MT. Olive oil decreases both oxidative stress and the production of arachidonic acid metabolites by the prostaglandin $\mathrm{G} / \mathrm{H}$ synthase pathway in rat macrophages. J Nutr. 2001;131(8):2145-2149.

35. Zheng Y, Chow SO, Boernert K. Direct crosstalk between cancer and osteoblast lineage cells fuels metastatic growth in bone via autoamplification of IL-6 and RANKL signaling pathways. J Bone Miner Res. 2011;19(9):1938-1949.

36. Papanicolaou DA, Wilder RL, Manolagas SC, et al. The pathophysiologic roles of interleukin- 6 in human disease. Ann Intern Med. 1998;128:127-137.

37. Lean JM, Davies JT, Fuller K et al. A crucial role for thiol antioxidants in estrogen-deficiency bone loss. J Clin Invest. 2003; 112(6):915-923.

38. Anderson GL, Judd HL, Kaunitz AM, et al. Effects of estrogen plus progestin on gynecologic cancers and associated diagnostic procedures: the Women's Health Initiative randomized trial. JAMA 2003;290(13):1739-1748
Clinical Interventions in Aging

\section{Publish your work in this journal}

Clinical Interventions in Aging is an international, peer-reviewed journal focusing on evidence-based reports on the value or lack thereof of treatments intended to prevent or delay the onset of maladaptive correlates of aging in human beings. This journal is indexed on PubMed Central, MedLine,

\section{Dovepress}

CAS, Scopus and the Elsevier Bibliographic databases. The manuscript management system is completely online and includes a very quick and fair peer-review system, which is all easy to use. Visit http://www.dovepress. com/testimonials.php to read real quotes from published authors. 